\title{
Rapid emergence of African swine fever virus variants with different numbers of a tandem repeat sequence in South Korea.
}

\author{
Seon-Hee Kim¹, Song-I Lee ${ }^{1}$, Hyun-Gi Jeong ${ }^{1}$, Jongchan Yoo ${ }^{2}$, Hyesung Jeong ${ }^{1}$, Yongjun \\ $\mathrm{Choi}^{1}$, Kidong Son ${ }^{1}$, and Jheong Weon-Hwa ${ }^{1}$ \\ ${ }^{1}$ National Institute of Environmental Research \\ ${ }^{2}$ Ministry of Environment
}

June 17,2020

\begin{abstract}
African swine fever virus variants with different numbers of a 10-bp tandem repeat were isolated in South Korea soon after being identified in wild boar. The short emergence periods and sympatric distributions within a narrow geographical region suggest that the variants were sporadically generated in the pre-existing viral population.
\end{abstract}

Rapid emergence of African swine fever virus variants with different numbers of a tandem repeat sequence in South Korea

Running Title: African swine fever virus variants in South Korea

Seon-Hee Kim ${ }^{1+}$, Song-I Lee ${ }^{1+}$, Hyun-Gi Jeong ${ }^{2}$, Jongchan Yoo ${ }^{3}$, Hye-Sung Jeong ${ }^{1}$, Yongjun Choi ${ }^{1}$, Kidong Son ${ }^{1}$, Weon-Hwa Jheong ${ }^{1 *}$

+ These authors contributed equally to this article.

${ }^{1}$ Biosafety Research Team, Environmental Health Research Department, National Institute of Environmental Research, Incheon, South Korea

${ }^{2}$ Nakdong River Environment Research Center, National Institute of Environmental Research, Daegu, South Korea

${ }^{3}$ Nakdong River Basin Environment Office, Ministry of Environment, Changwon, South Korea

*Corresponding author

Address for correspondence: Weon-Hwa Jheong, Biosafety Research Team, Environmental Health Research Department, National Institute of Environmental Research, 22689, Incheon, South Korea. Email:purify@korea.kr: Tel: +82-32-560-7140, Fax: +82-32-568-2036

\section{Summary}

African swine fever virus variants with different numbers of a 10-bp tandem repeat were isolated in South Korea soon after being identified in wild boar. The short emergence periods and sympatric distributions within a narrow geographical region suggest that the variants were sporadically generated in the pre-existing viral population. 
Keywords : African swine fever virus; tandem repeat; intergenic region variant; replication slippage; wild boar; South Korea

\section{Introduction}

African swine fever (ASF) is a highly contagious disease occurring in domestic pigs and wild suids, and leads to serious haemorrhage and nearly $100 \%$ mortality. ASF is caused by infection with African swine fever virus (ASFV; family: Asfarviridae, genus: Asfivirus ) (Dixon et al., 2020). Since its original description in the 1920s in Kenya, this viral disease has been highly prevalent in African and European countries. In Asia, ASF was first reported in a farm neat Shenyang City in Liaoning province in China, in August 2018 (Zhou at al., 2018). Thereafter, it quickly spread to other countries in Southeast Asia including Myanmar, Laos, Vietnam, and the Philippines, as well as nearly all provinces in mainland China, in 2019 (Lu et al., 2020). ASF outbreaks have also been described in North and South Koreas in the same year (Kim H. J. et al., 2020; Kim S. H. et al., 2020).

Despite the high mortality rates and socio-economic impacts of ASF, no vaccines or therapeutic agents are available for controlling its outbreak or its effective treatment (Dixon et al., 2020). Therefore, studies on the routes and patterns of ASF transmission as well as its early detection are urgently needed. Molecular epidemiology approaches using polymorphic DNA sequences can provide insight into the spatiotemporal patterns of disease transmission throughout the areas in which ASF is prevalent. The genomic DNA of ASFV shows a low evolution rate. Nevertheless, multiple sites show inter-genomic polymorphisms, particularly those containing short tandem repeats (STRs), which can be selected as informative markers in epidemiological investigations (Goller et al, 2015; Nix et al, 2006).

After ASFV was first isolated from a wild boar in October 2, 2019 in South Korea (Kim S. H. et al., 2020), we conducted a surveillance programme for wild boars in the relevant areas by the National Institute of Environmental Research (NIER). Genotypes of ASFV DNAs obtained during these surveys were investigated via polymerase chain reaction (PCR) and nucleotide sequencing to trace viral transmission in the wild boar population and monitor the probable emergence of viral variants.

\section{Material and Methods}

During the nationwide comprehensive monitoring of wild boars from October 2 to December 30, 2019, 56 viral isolates were collected from whole blood or tissue of wild boar carcasses in the border area covering Paju and Yeoncheon counties of Gyeonggi Province and Cheorwon county of Gangwon Province by NIER (Fig. 1). The positive rate was highest in Paju (Table 1). Partial segments of B646L (p72) and EP402R(CD2v) as well as an intergenic region (IGR) between I73R andI329L were amplified from each of the viral DNAs by PCR using specific primer sets, as described previously (Kim S. H. et al., 2020). The PCR products were subject to automated paired-end sequencing reactions and the resulting contig sequences were used in phylogenetic analyses together with their homologues retrieved from the GenBank database (neighbour-joining algorithm with the MEGA 6.0 software).

\section{Results and Discussion}

All partial B646L and EP402R sequences of the ASFV isolates were identical to those of the original Korean isolate, Korea/19S804/wb/2019 (GenBank accession nos. MN817977 and MN817978). These sequences were categorised into the genotype II (Fig. 2A) and serogroup 8 (Fig. 2B) groups, respectively. Most IGR fragments also showed $100 \%$ sequence identity to the corresponding region of the Korea/19S804/wb/2019 isolate (GenBank accession no. MN817979), except for those of two isolates collected in Paju (Korea/19S3965/wb/2019 on December 3 and Korea/19S5464/wb/2019 on December 30). The IGR fragment contained an STR (5'-GGAATATATA-3'), with a repeat time varying in or among ASFV populations (Goller et al., 2015; Nix et al., 2006). The 10-bp STR was inserted three times in the corresponding regions of the major Korean ASFV isolates from the wild boar (IGR variant II), similar to those in the Russia/Volgograd/wb/2014 (GenBank accession no. KP137637), Belgium/Etalle/wb/2018 (GenBank accession no. MH998359.1), and China/2018/Domestic pig (GenBank accession no. MH 735144) strains. The 
genome of another Korean isolate from a domestic pig (Korea/2019/Domestic pig, GenBank accession no. MN603969 [Kim H. J. et al., 2020]) also belonged to the variant II group. On the other hand, the nucleotide stretch was repeated two and four times in the genomes of the Korea/19S3965/wb/2019 (IGR variant I) and Korea/19S5464/wb/2019 (IGR variant III) isolates, respectively (Table 2).

Because the genomes of DNA viruses, including ASFV, show relatively low evolutionary divergence, few informative molecular markers have been detected within the genic regions of the viral genomes, as observed for the by B646L and EP402R sequences in this study. However, the lengths of genomic STR are readily expanded or contracted during DNA replication largely by slipped strand mispairing (slippage mechanism). If the affected STR is in an intergenic noncoding region, the allelic variant can be fixed in the population because of the low levels of purifying selection (Gemayel et al., 2010). ASFV strains with variant IGR genotypes have been discovered in Russia (Goller et al., 2015) and China (Ge et al., 2019; Li et al., 2019). However, unlike the IGR variants in these countries, those identified in this study were sympatrically distributed with the pre-existing type in a small county (Fig. 1B). The time intervals for their emergence were also very short (approximately two and three months after the first outbreak in wild boars). Taken together, these facts may suggest that the Korean IGR variants I and III were sporadically generated rather than being independently transported from other countries through replication error in the pre-existing IGR variant II population.

In this study, we identified ASFV variants with different genotypes collected during the comprehensive survey of wild boars in small counties of South Korea surrounding the original ASF outbreak point. Considering the short emergence periods of less than three months and sympatric distributions within a narrow geographical region, these variant strains are likely to have spontaneously emerged in the local viral population through a molecular mechanism(s) such as replication slippage.

The probable transmission routes of ASF can be predicted by analysing the spatiotemporal distributions of ASFV with distinct IGR genotypes (Goller et al., 2015). Therefore, the polymorphic STR was suggested as an informative marker to discriminate closely related ASFV strains (Ge et al., 2019). Currently, we have no evidence supporting clonal expansion of these variants in the relevant region, which may be because of their recent emergence. Surveillance of wild boars will be continued until the viral disease is eliminated. If simultaneous propagation of these IGR variants is observed in the near future, our data will provide a highly informative genetic marker for molecular epidemiological approaches to trace both local and global transmission of ASFV.

\section{Acknowledgements}

This study was funded (Grant Number: NIER-2019-01-01-006) by the National Institute of Environmental Research (NIER), Ministry of Environment, Republic of Korea.

\section{Author Contributions}

This manuscript was written by S-H Kim and W-H Jheong: experiment and data analysis were performed by S-H Kim, S-I Lee, H-G Jeong, J-Yoo, K Son, H-S-Jeong and study was designed by S-H Kim and W-H Jheong. Isolate partial sequence data from this study were deposited in GenBank with the accession numbers MT300320 to MT300325.

\section{Ethical approval}

Not applicable

\section{Conflict of Interest}

The authors declare no competing interests.

\section{Availability of data}

The data that support the finding of this study are available from the corresponding author upon reasonable request. 


\section{References}

Dixon, L. K., Stahl, K., Jori, F., Vial, L., \& Pfeiffer, D. U. (2020). African swine fever epidemiology and control. Annual Review of Animal Biosciences, 15, 221-246.https://doi.org/10.1146/annurev-animal021419-083741

Ge, S., Liu, Y., Li, L., Wang, Q., Li, J., Ren, W., Liu, C., Bao, J., Wu, X., \& Wang, Z. (2019). An extra insertion of tandem repeat sequence in African swine fever virus, China, 2019. Virus Genes, 55, 843-847. doi:https://doi.org/10.1007/s11262-019-01704-9

Gemayel, R., Vinces, M. D., Legendre, M., \& Verstrepen, K. J. (2010). Variable tandem repeats accelerate evolution of coding and regulatory sequences. Annual Review of Genetics, 44, 445477.https://doi.org/10.1146/annurev-genet-072610-155046

Goller, K. V., Malogolovkin, A. S., Katorkin, S., Kolbasov,D., Titov, I., Höper, D., Beer, M., Keil., G. M., Portugal, R., \& Blome, S. (2015). Tandem repeat insertion in African swine fever virus, Russia, 2012. Emerging Infectious Diseases, 21, 731-732. doi:https://dx.doi.org/10.3201\%2Feid2104.141792

Kim, H. J., Cho, K. H., Lee, S. K., Kim, D. Y., Nah, J. J., Kim, H. J., Kim, H. J., Hwang, J. Y., Sohn, H. J., Choi, J. G., Kang, H. E., \& Kim, Y. J. (2020). Outbreak of African swine fever in South Korea, 2019. Transboundary and Emerging Diseases, 67, 473-475.https://doi.org/10.1111/tbed.13483

Kim, S. H., Kim, J., Son, K., Choi, Y., Jeong, H. S., Kim, Y. K., Park, J. E., Hong, Y. J., Lee, S. I., Wang, S. J., Lee, Y. S., Kim, W. M., \& Jheong, W. H. (2020). Wild boar harbouring African swine fever virus in the demilitarized zone in South Korea, 2019. Emerging Microbes \& Infections, 9, 628630.https://doi.org/10.1080/22221751.2020.1738904

Li, L., Ren, Z., Wang, Q., Ge, S., Liu, Y., Liu, C., Liu, F., Hu, Y., Li, J., Bao, J., Ren, W., Zhang, Y., Xu, T., Sun, C., Li, L., Wang, S., Fan, X., Wu, Z., Huang, B., Guo, H., et al. (2019). Infection of African swine fever in wild boar, China, 2018. Transboundary and Emerging Diseases, 66, 13951398.https://doi.org/10.1111/tbed.13114

Lu, G., Pan, J., \& Zhang, G. (2020). African swine fever virus in Asia: Its rapid spread and potential threat to unaffected countries. Journal of Infection, 80, 350-371.https://doi.org/10.1016/j.jinf.2019.11.011

Nix, R. J., Gallardo, C., Hutchings, G., Blanco, E., \& Dixon, L. K. (2006). Molecular epidemiology of African swine fever virus studied by analysis of four variable genome regions. Archives of Virology, 151, 2475-2494.https://doi.org/10.1007/s00705-006-0794-z

Zhou X,, Li N., Liu Y., Miao F., Chen T., Zhang S., Cao P., Li X., Tian K., Qiu HJ., \& Hu R (2018). Emergence of African Swine Fever in China. Transboundary and Emerging Diseases, 65, 14821484.https://doi.org/10.1111/tbed.12989

\section{Tables}

Table 1. Summary of results for wild boars examined for ASFV infection in 2019 in South Korea

\begin{tabular}{llll}
\hline County & No. of wild boars tested & $\begin{array}{l}\text { No. of infected wild } \\
\text { boars }(\%)\end{array}$ & $\begin{array}{l}\text { No. of positive whole } \\
\text { blood/tissue samples }\end{array}$ \\
\hline Yeoncheon & 302 & $19(6.3)$ & $4 / 15$ \\
Cheorwon & 473 & $17(3.4)$ & $6 / 14$ \\
Paju & 117 & $20(17.1)$ & $8 / 9$ \\
Other areas & 3782 & $0(0)$ & - \\
Total & 4674 & $56(1.0)$ & $18 / 38$ \\
\hline
\end{tabular}

Table 2. Comparison of intergenic region (IGR) sequences betweenI73R and I329L encompassing a short 
tandem repeat (STR).

\begin{tabular}{|c|c|c|}
\hline GenBank No. & Strain & Partial nucleotide sequence encompassing the STR in IGR between \\
\hline MT300324 & Korea/19S3965/wb/2019 & GCAATAAATAACAAGTATATA GGAATATATAGGAATATATA- \\
\hline FR682485 & Georgia 2007/1 & GCAATAAATAACAAGTATATA GGAATATATAGGAATATATA- \\
\hline KY982843 & Russia/Irkutsk/2017/Domestic pig & GCAATAAATAACAAGTATATA GGAATATATAGGAATATATA \\
\hline MK189457 & China/jilin/2018/boar & GCAATAAATAACAAGTATATA GGAATATATAGGAATATATA \\
\hline MG939584 & Poland/Pol16/2016/wb & GCAATAAATAACAAGTATATA GGAATATATAGGAATATATA \\
\hline KP137637 & Russia/Volgograd/wb/2014 & GCAATAAATAACAAGTATATA GGAATATATAGGAATATATA \\
\hline MH998359 & Belgium/Etalle/wb/2018 & GCAATAAATAACAAGTATATA GGAATATATAGGAATATATA \\
\hline MH735144 & China/2018/Domestic pig & GCAATAAATAACAAGTATATA GGAATATATAGGAATATATA \\
\hline MN603969 & Korea/2019/Domestic pig & GCAATAAATAACAAGTATATA GGAATATATAGGAATATATA \\
\hline MN817979* & Korea/19S804/wb/2019 & GCAATAAATAACAAGTATATA GGAATATATAGGAATATATA \\
\hline MK670729 & China/Guangxi/2019/Domestic pig & GCAATAAATAACAAGTATATA GGAATATATAGGAATATATA \\
\hline MT300325 & Korea/19S5464/wb/2019 & GCAATAAATAACAAGTATATA GGAATATATAGGAATATATA \\
\hline
\end{tabular}

* The nucleotide sequences obtained in this study (bold sequences) were compared to those of other African swine fever virus (ASFV) strains representing each IGR genotype. Of the 56 ASFV sequences, 54 sequences, excluding the 19S3965 and 19S5464 isolates, were represented by the pre-existing Korea/19S804/wb/2019 isolate (marked with asterisk), showing 100\% identity. The 10-bp STR unit (5'-GGAATATATA-3') is underlined.

Figure Legends :

Figure 1. Global (A) and locality (B) maps showing the collection sites of African swine fever virus from wild boars in South Korea from October 2 to December 30, 2019. Local area in Paju county where the IGR variants and pre-existing strain were isolated (dotted box in panel A) are magnified in panel B. Dot marked with an arrow in the inset map of panel A indicates the place where the first African swine fever case was detected in a wild boar in South Korea. The collection sites of IGR variants I, II, and III in Paju county are indicated by a triangle, dot, and square, respectively, in panel B.

Figure 2. Phylogenetic analyses of partial B646L (A) andEP402R (B) sequences of African swine fever virus isolates obtained in this study. The neighbour-joining trees were constructed with MEGA 6 based on the Kimura 2-parameter model. Numerals on branching nodes indicate the bootstrap values obtained with 1,000 replicates $(>50 \%)$. The sequences obtained in this study are distinguished by bold letters, while the phylogenetic positions of 54 sequences other than those of 19S3965 and 19S5464 isolates are represented by that of the pre-existing Korea/19S804/wb/2019 isolate (marked with asterisk).

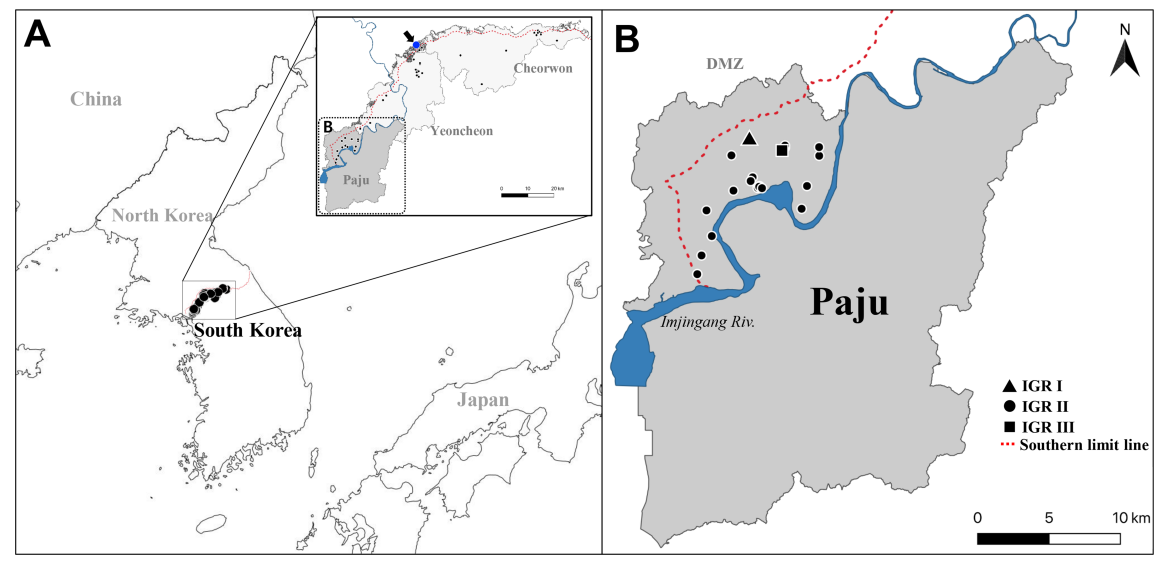


A

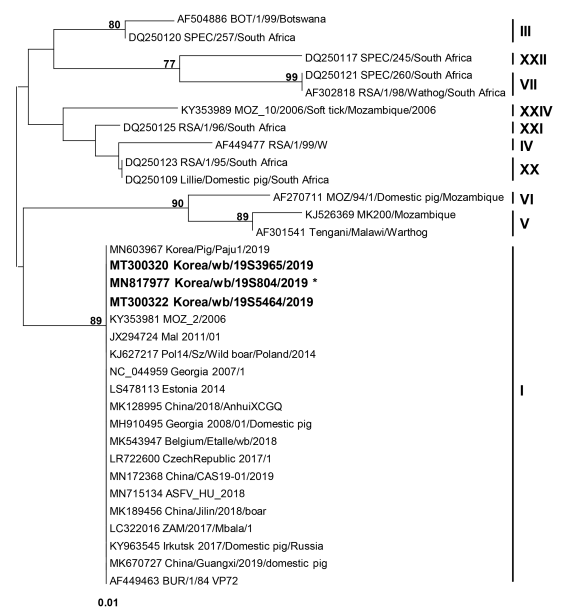

B

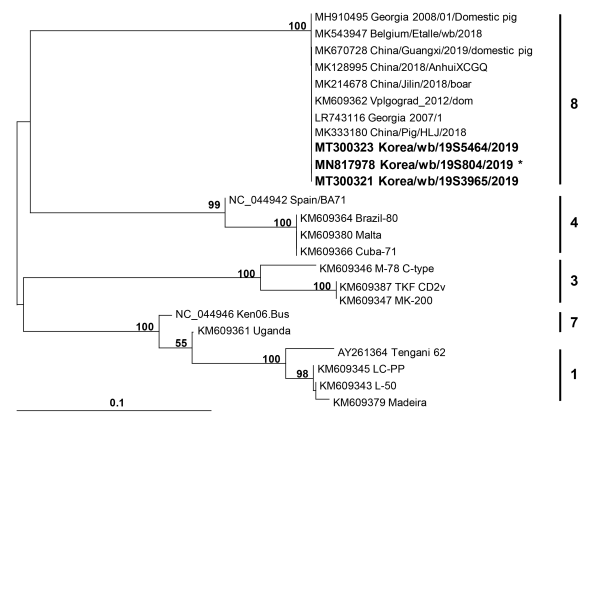

